\title{
On a probability inequality of van Dam
}

\section{Zi-zong Yan* and Yue-mei Zhang}

"Correspondence: zzyan@yangtzeu.edu.cn School of Information and Mathematics, Institute of Applied Mathematics, Yangtze University, Jingzhou, Hubei, China

\begin{abstract}
This note derives an interesting probability inequality between the expectation of a conditional variance and the variance of a conditional expectation for a function of two independent random variables. In the special case of finite discrete random variables, the inequality coincides with an inequality by Feng and Tonge (2000), which extends a result by van Dam (1998).
\end{abstract}

MSC: 26E60

Keywords: probability inequality; random variable; van Dam's inequality

Let $T$ be a continuous random variable having the probability density function $\phi$ defined on $R$. By definition

$$
E(T)=\int_{-\infty}^{\infty} t \phi(t) d t
$$

is the expectation of $T$, and

$$
\sigma^{2}(T)=\int_{-\infty}^{\infty}(t-E(T))^{2} \phi(t) d t
$$

is the variance $\sigma^{2}(T)$.

Let $X, Y$ be two independent random variables with known distribution having the probability density function $\phi_{1}(x)$ and $\phi_{2}(y)$, respectively. Then the joint probability density function of $X$ and $Y$ is $\phi_{1}(x) \phi_{2}(y)$.

Let another random variable $Z$ be a function of $X$ and $Y$

$$
Z=f(X, Y)
$$

where $f(\cdot, \cdot) \in L^{2}\left(R^{2}\right)$. Then the expectation of $Z$ is

$$
E(Z)=\int_{-\infty}^{\infty} \int_{-\infty}^{\infty} f(x, y) \phi_{1}(x) \phi_{2}(y) d x d y
$$

and the conditional probability density functions of $Z$, given the occurrence of the value $x$ of $X$ and $y$ of $Y$, are equal to $\phi_{2}(y)$ and $\phi_{1}(x)$, respectively, such that the conditional expectations of $Z$ are equal to

$$
E(Z \mid X)=\int_{-\infty}^{\infty} f(x, y) \phi_{2}(y) d y, \quad E(Z \mid Y)=\int_{-\infty}^{\infty} f(x, y) \phi_{1}(x) d x .
$$

(0) 2015 Yan and Zhang; licensee Springer. This is an open access article distributed under the terms of the Creative Commons Attribution License (http://creativecommons.org/licenses/by/2.0), which permits unrestricted use, distribution, and reproduction in any medium, provided the original work is properly cited. 
Furthermore, we have

$$
\begin{aligned}
& \begin{aligned}
E(E(Z \mid X)) & =\int_{-\infty}^{\infty}\left(\int_{-\infty}^{\infty} f(x, y) \phi_{2}(y) d y\right) \phi_{1}(x) d x \\
= & \int_{-\infty}^{\infty} \int_{-\infty}^{\infty} f(x, y) \phi_{2}(y) \phi_{1}(x) d x d y=E(Z), \\
E(Z \cdot E(Z \mid X)) & =\int_{-\infty}^{\infty} \int_{-\infty}^{\infty} f(x, y)\left(\int_{-\infty}^{\infty} f(x, t) \phi_{2}(t) d t\right) \phi_{1}(x) \phi_{2}(y) d x d y \\
& =\int_{-\infty}^{\infty}\left(\int_{-\infty}^{\infty} f(x, y) \phi_{2}(y) d y\right)^{2} \phi_{1}(x) d x=E\left([E(Z \mid X)]^{2}\right),
\end{aligned}
\end{aligned}
$$

and

$$
\begin{aligned}
E & (E(Z \mid X) \cdot E(Z \mid Y)) \\
& =\int_{-\infty}^{\infty} \int_{-\infty}^{\infty}\left(\int_{-\infty}^{\infty} f(s, y) \phi_{1}(s) d s\right)\left(\int_{-\infty}^{\infty} f(x, t) \phi_{2}(t) d t\right) \phi_{1}(x) \phi_{2}(y) d x d y \\
& =\int_{-\infty}^{\infty} \int_{-\infty}^{\infty} f(s, y) \phi_{1}(s) \phi_{2}(y) d s d y \int_{-\infty}^{\infty} \int_{-\infty}^{\infty} f(x, t) \phi_{1}(x) \phi_{2}(t) d x d t=[E(Z)]^{2} .
\end{aligned}
$$

Shortly,

$$
\begin{aligned}
& E(E(Z \mid X))=E(Z), \\
& E(Z \cdot E(Z \mid X))=E\left([E(Z \mid X)]^{2}\right), \\
& E(E(Z \mid X) \cdot E(Z \mid Y))=[E(Z)]^{2} .
\end{aligned}
$$

Theorem 1 Let $X, Y$ be two independent random variables and $Z=f(X, Y)$ be a function of the random variables $X$ and $Y$. Then

$$
E\left(\sigma^{2}(Z \mid X)\right) \leq \sigma^{2}(E(Z \mid Y))
$$

Proof For convenience, we set $Z_{1}=E(Z \mid X)$ and $Z_{2}=E(Z \mid Y)$. It follows from (3) that

$$
E\left(Z-Z_{1}-Z_{2}\right)=-E(Z)
$$

Since $\sigma^{2}\left(Z-Z_{1}-Z_{2}\right)=E\left(\left[Z-Z_{1}-Z_{2}\right]^{2}\right)-\left[E\left(Z-Z_{1}-Z_{2}\right)\right]^{2} \geq 0$,

$$
[E(Z)]^{2} \leq E\left(\left[Z-Z_{1}-Z_{2}\right]^{2}\right)
$$

From (4) and (5), we have

$$
E\left(Z Z_{1}\right)=E\left(Z_{1}^{2}\right), \quad E\left(Z Z_{2}\right)=E\left(Z_{2}^{2}\right)
$$

and

$$
E\left(Z_{1} Z_{2}\right)=[E(Z)]^{2}
$$


Therefore, we have

$$
\begin{aligned}
E( & {\left.\left[Z-Z_{1}-Z_{2}\right]^{2}\right) } \\
& =E\left(Z^{2}+Z_{1}^{2}+Z_{2}^{2}-2 Z Z_{1}-2 Z Z_{2}+2 Z_{1} Z_{2}\right) \\
& =E\left(Z^{2}\right)+E\left(Z_{1}^{2}\right)+E\left(Z_{2}^{2}\right)-2 E\left(Z_{1}^{2}\right)-2 E\left(Z_{2}^{2}\right)+2[E(Z)]^{2} \\
& =E\left(Z^{2}\right)-E\left(Z_{1}^{2}\right)-E\left(Z_{2}^{2}\right)+2[E(Z)]^{2} .
\end{aligned}
$$

Combining with (7), we get

$$
E\left(Z_{1}^{2}\right)-[E(Z)]^{2} \leq E\left(Z^{2}\right)-E\left(Z_{2}^{2}\right)
$$

which is equivalent to the desired inequality.

When $X$ and $Y$ are two finite discrete random variables, a discrete version of (6) is as follows:

$$
\sum_{i=1}^{m}\left(\sum_{j=1}^{n} a_{i j} q_{j}\right)^{2} p_{i}+\sum_{j=1}^{n}\left(\sum_{i=1}^{m} a_{i j} p_{i}\right)^{2} q_{j} \leq\left(\sum_{i=1}^{m} \sum_{j=1}^{n} a_{i j} p_{i} q_{j}\right)^{2}+\sum_{i=1}^{m} \sum_{j=1}^{n} a_{i j}^{2} p_{i} q_{j},
$$

where the nonnegative real numbers $p_{i}(1 \leq i \leq m)$ and $q_{j}(1 \leq j \leq n)$ satisfy $\sum_{i=1}^{m} p_{i}=1$ and $\sum_{j=1}^{n} q_{i}=1, A=\left(a_{i j}\right)$ is a real $m \times n$ matrix. This discrete version is given by Feng and Tonge [1].

If we put $p_{i}=\frac{1}{m}, i=1,2, \ldots, m$ and $q_{j}=\frac{1}{n}, j=1,2, \ldots, \frac{1}{n}$, then (8) becomes the following van Dam inequality:

$$
\sum_{i=1}^{m}\left(\sum_{j=1}^{n} a_{i j}\right)^{2}+\sum_{j=1}^{n}\left(\sum_{i=1}^{m} a_{i j}\right)^{2} \leq\left(\sum_{i=1}^{m} \sum_{j=1}^{n} a_{i j}\right)^{2}+\sum_{i=1}^{m} \sum_{j=1}^{n} a_{i j}^{2}
$$

van Dam [2] applied his inequality to pose a related problem on the maximum irregularity of a directed graph with prescribed number of vertices and arcs.

In the special case of $(0,1)$-matrices, the inequality (9) reduces to the following Khinchin-type inequality:

$$
m \sum_{i=1}^{m} r_{i}^{2}+n \sum_{j=1}^{n} c_{j}^{2} \leq \sigma^{2}+m n \sigma
$$

where $r_{i}, c_{i}$, and $\sigma$ denote row sums, column sums, and entries summing of an $m \times n$ $(0,1)$ matrix, respectively, as presented by Matúš and Tuzar [3]. This inequality is an improvement (in the nonsquare case) of a result by Khinchin [4], who proved that $l \sum_{i=1}^{m} r_{i}^{2}+$ $l \sum_{i=1}^{m} c_{i}^{2} \leq \sigma^{2}+l^{2} \sigma$, where $l=\max \{m, n\}$. Khinchin [5] applied his inequality to prove a surprising number theoretic result.

Recently, Yan [6] presented another extension of (9). It is natural to ask whether the analog of (6) holds or not for three independent random variables. We have been unable to prove (or disprove) it. 
Competing interests

The authors declare that they have no competing interests.

Authors' contributions

All authors contributed equally to the writing of this paper. All authors read and approved the final manuscript.

\section{Acknowledgements}

The many suggestions and detailed corrections of anonymous referees are gratefully acknowledged. Supported by the National Natural Science Foundation of China (11201039; 61273179).

Received: 3 July 2014 Accepted: 31 March 2015 Published online: 11 April 2015

\section{References}

1. Feng, BQ, Tonge, F: A Cauchy-Khinchine integral inequality. Linear Algebra Appl. 433, 1024-1030 (2000)

2. van Dam, ER: A Cauchy-Khinchine matrix inequality. Linear Algebra Appl. 280, 163-172 (1998)

3. Matúš, F, Tuzar, A: Short proofs of Khintchine-type inequalities for zero-one matrices. J. Comb. Theory, Ser. A 59, 155-159 (1992)

4. Khinchin, A: Über eine Ungleichung. Mat. Sb. 39, 35-39 (1932)

5. Khinchin, A: Über ein metrisches Problem der additieven Zahlentheorie. Mat. Sb. 40, 180-189 (1933)

6. Yan, Z: A Cauchy-Khinchin-van Dam matrix inequality. Linear Multilinear Algebra 59(7), 825-879 (2011)

\section{Submit your manuscript to a SpringerOpen ${ }^{\circ}$ journal and benefit from:}

- Convenient online submission

Rigorous peer review

- Immediate publication on acceptance

- Open access: articles freely available online

- High visibility within the field

- Retaining the copyright to your article 